\title{
Introducción a la robótica con actividades de construcción, programación y competición de robots
}

\author{
Francisco A. Candelas, Fernando Torres, Santiago T. Puente \\ Departamento de Física, Ingeniería de Sistemas y Teoría de la señal, Universidad de Alicante \\ \{francisco.candelas, fernando.torres, santiago.puente\}@ua.es
}

\begin{abstract}
Resumen
Este documento describe la planificación y el desarrollo de unas prácticas de laboratorio para la introducción de la robótica, así como algunas experiencias y resultados obtenidos de ellas. Las prácticas se han llevado a cabo en la asignatura "Introducción a la Ingeniería Robótica" durante el curso académico 2015-2016. Ésta es una asignatura obligatoria del primer curso del nuevo Grado en Ingeniería Robótica que ha empezado a impartirse en la Universidad de Alicante en el citado curso, y en la cual muchos alumnos tienen el primer contacto con la robótica. Por ello, el principal objetivo de las prácticas de laboratorio es introducir los conceptos básicos relacionados con el funcionamiento, diseño y programación de robots, a la vez que los alumnos desarrollan sus habilidades para resolver problemas típicos de ingeniería y se enfrentan a problemas prácticos que se dan en la construcción de un robot. Durante el curso, cada equipo de alumnos tiene que crear un pequeño robot móvil que sea capaz de resolver determinadas situaciones sobre un circuito propuesto, usando para ello los sensores y accionamientos adecuados. Además, al final de la asignatura, los equipos deben completar el circuito con sus robots, considerando aspectos como el tiempo y los fallos. Para construir los robots se ha escogido un kit comercial con piezas robustas y cuyo controlador está basado en Arduino/Genuido.
\end{abstract}

Palabras Clave: Robótica, prácticas de laboratorio, competición, Arduino/Genuino.

\section{INTRODUCCIÓN}

El trabajo descrito aquí se enmarca dentro de la asignatura Iniciación a la Ingeniería Robótica, que se imparte como asignatura obligatoria en el segundo semestre del primer curso del Grado en Ingeniería Robótica. Este nuevo grado se ha ofertado en la Universidad de Alicante por primera vez en el curso 2015-16, y por ello es la primera vez que se oferta la asignatura [11]. La asignatura tiene 6 créditos ECTS, que incluyen 30 horas de teoría, 15 horas de prácticas de laboratorio y 15 horas de visitas, además de 90 horas de trabajo fuera del aula. Las clases de teoría y las prácticas de laboratorio se complementan con visitas a empresas o laboratorios de investigación donde se utilizan robots para diferentes aplicaciones. Durante el curso 2015-16, ha habido 62 alumnos matriculados en la asignatura, que se corresponden con los matriculados en el primer curso.

Esta contribución se centra en describir las actividades para las prácticas de laboratorio que se han planificado y propuesto a los alumnos. Para elaborar las actividades antes de empezar la asignatura, partimos de la idea de que los alumnos deberían diseñar y construir sus propios robots a partir de algún conjunto de piezas o kit de construcción para robótica. También se pensó que sería mejor usar piezas conectadas que permitiesen un montaje clásico en base a tornillos, en vez de un kit de construcción estándar, porque de ese modo los montajes realizados serían más robustos, y sobre todo, más similares a los robots reales.

Otro aspecto importante que hubo que considerar fue el hecho de que la asignatura Iniciación a la Ingeniería Robótica es el primer contacto de muchos alumnos con el mundo de la robótica. Además, muchos de los alumnos son novatos en programación, ya que ellos todavía no han acabado la primera asignatura sobre programación que hay en el Grado, porque ésta se imparte en el segundo cuatrimestre a la vez que Iniciación a la Ingeniería Robótica. De este modo, el kit escogido para construir los robots debería ser fácil de programar.

Para la programación de robots en cursos de introducción, la plataforma Arduino (ahora también conocida como Genuino fuera de USA) es probablemente la mejor opción en la actualidad. La principal ventaja de esta plataforma es que su puesta en marcha y aprendizaje son rápidos. Así, los alumnos pueden centrarse en el desarrollo y programación de los algoritmos del control que hacen funcionar el robot con las especificaciones dadas. Además, el IDE (Integrated Development Environment) que se utiliza para programar todas las placas de Arduino es software abierto y gratuito, además de fácil de poner en marcha y de usar. Arduino se programa en $\mathrm{C}++$, lo cual permite a los 
usuarios más avanzados desarrollar programas complejos a la vez que los usuarios noveles pueden comenzar con programas muy simples. Otro aspecto destacable de la plataforma Arduino es la gran cantidad de documentación que se puede encontrar sobre ella, que abarca desde la documentación básica ofrecida en el sitio web oficial, hasta libros de texto completos para diferentes campos de aplicación $[1][2][13]$.

Un hecho que muestra el éxito de la plataforma Arduino es la cantidad de cursos específicos sobre ella que se están ofreciendo en la actualidad, algunos de ellos patrocinados por instituciones relevantes, como es el caso del curso organizado por CEA (Comité Español de Automática) [4]. Cursos como éste no solamente están enfocados a personas con un interés especial en la electrónica o la robótica, sino también a profesores que quieren usar la plataforma en la educación.

Todo lo comentado en los párrafos anteriores ha contribuido a que Arduino se haya extendido ampliamente en el contexto de la educación, tanto a nivel de bachillerato como de grado. Arduino es especialmente popular en la educación de electrónica, control automático o robótica [3][9][10][12].

Considerando los hechos descritos sobre la plataforma Arduino, así como los trabajos y resultados de otros investigadores [3][5][9][10], tuvimos muy claro desde el principio que habría que elegir un kit de construcción para los robots cuyo controlador estuviese basado en Arduino.

El resto de esta contribución se centra en describir el kit de construcción que fue escogido para las prácticas de laboratorio, así como las actividades que se proponen a los alumnos. Estos aspectos se tratan en las secciones II y III respectivamente. La sección IV comenta los resultados obtenidos en relación al interés y aprendizaje de los alumnos. Finalmente, la sección IV describe las principales conclusiones que se han extraído del trabajo realizado.

\section{KITS DE CONSTRUCCIÓN}

Como se mencionó en la introducción, decidimos emplear un kit de construcción que permitiese a los alumnos diseñar y construir robots robustos y fáciles de programar. Entre las diferentes opciones disponibles en el mercado, finalmente decidimos adquirir varias unidades del Ultimate Robot Kit de la casa Makeblock [6], cuyo aspecto se muestra en la Figura 1.

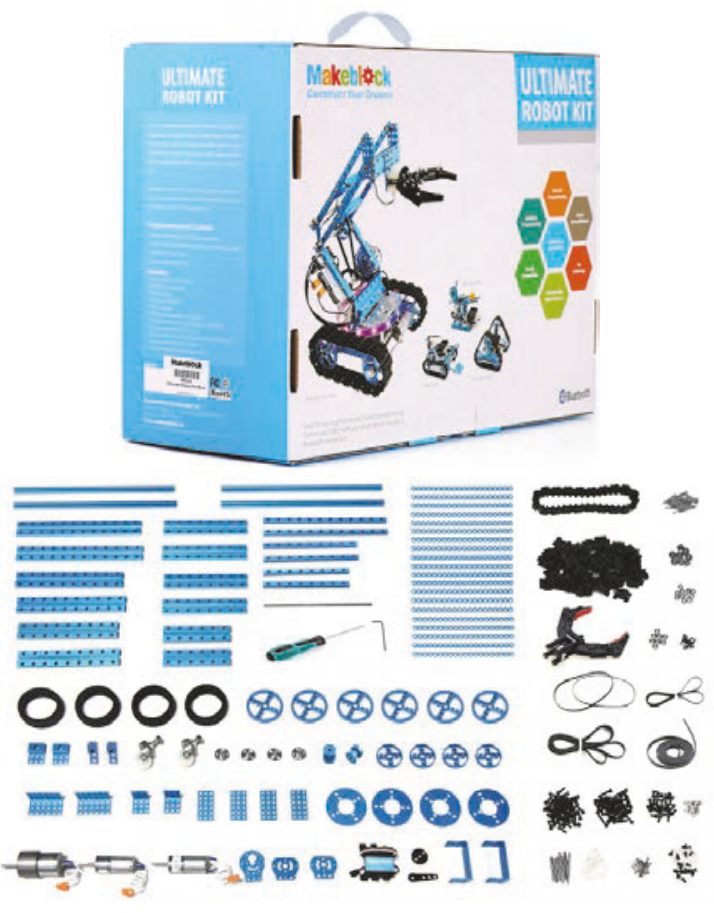

Figura 1: Ultimate Robot Kit de la casa Makeblock.

Makeblock vende diferentes kits para robótica, así como piezas individuales, que se caracterizan por emplear piezas de aluminio fuertes y ligeras, que se montan mediante tornillos $\mathrm{y}$ tuercas. Estas características contrastan con la mayoría de alternativas existentes que utilizan piezas de plástico, o cuyos montajes se pueden desarmar involuntariamente por no emplear otra sujeción aparte del propio encaje de las piezas (como Lego, Fischertechnik, VEX Super Kit). Otras opciones también fueron descartadas por ser demasiado básicas al estar enfocadas a un público de poca edad (Tinker Bots, JJBots, OLLO Explorer), o porque sus kits están centrados en un tipo de robot y son poco flexibles para crear diseños propios (Gears IDS, Multiplo).

La variedad de piezas del Ultimate Robot Kit permite al usuario construir una amplia variedad de pequeños robots, que pueden llegar a ser tener cierta complejidad. Entre los componentes que se incluyen en el kit (ver la Figura 1) hay 3 motores CC con reductora, una piza con cierre gradual mediante otro motor CC, varios engranajes y poleas, diferentes ruedas y cadenas de tanque, pulsadores, un sensor de distancia por ultrasonidos, un detector de marcas en el suelo mediante infrarrojos, un módulo de comunicación Bluetooth, un detector de sonido, una tira de LEDs RGB y un módulo controlador. El kit también incluye las herramientas básicas para el montaje. De este modo, se puede construir desde un sencillo robot con tres ruedas, hasta un robot que tenga un brazo grúa con una pinza capaz de agarrar y desplazar objetos. 


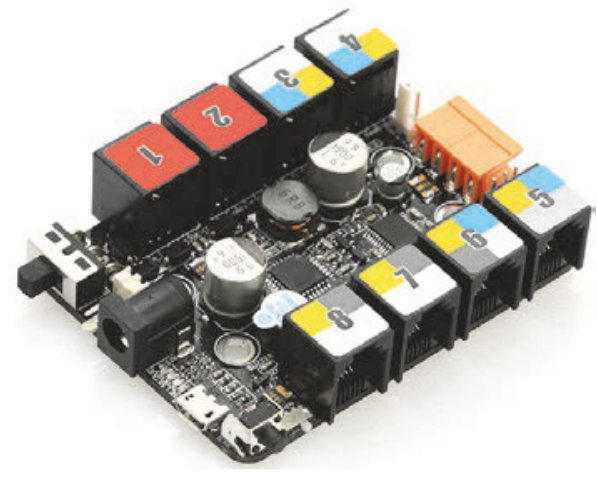

Figura 2: Placa del controlador Orion.

La última versión del controlador que incluye el kit se denomina Orion, y está basado en un Arduino UNO, siendo $100 \%$ compatible con la plataforma Arduino [7]. La placa del controlador Orion, cuyo aspecto se puede ver en la Figura 2, se diferencia de una de Arduino básicamente en dos aspectos. Primero, Orion dispone de 8 conectores RJ-45 hembra para el cableado con los módulos sensores, amplificadores de motores y de comunicación. También todos los módulos electrónicos que ofrece Makeblock emplean el mismo conector, lo que hace que la interconexión sea muy rápida y sencilla mediante los cables planos con conexión RJ-45 disponibles en varias longitudes, sin necesidad de usar cables individuales o soldaduras. Segundo, Orion incluye electrónica adicional como dos amplificadores para motores CC, un zumbador y un interruptor de encendido. Cabe destacar que todos los conectores e interruptores son fácilmente accesibles en los bordes de la placa.

Puesto que el controlador Orion es compatible con Arduino, éste puede ser programado con el IDE estándar de Arduino. El controlador también se puede programar mediante MBlock, un lenguaje gráfico basado en Scratch 2.0 [8]. Pero esta segunda opción es más adecuada para escuelas, mientras para las prácticas de Iniciación a la Ingeniería Robótica, donde se forman futuros ingenieros, es más adecuada la programación en $\mathrm{C}++$ con el IDE.

Makeblock proporciona gratuitamente una biblioteca de clases para el IDE de Arduino, la cual permite crear objetos que encapsulan el acceso y la gestión de los distintos módulos que se pueden conectar al controlador, y esto facilita mucho el acceso a sensores y accionamientos desde el programa.

Considerando los objetivos que se planteaban en la introducción para las prácticas de laboratorio, y las características que ofrecen los kits de Makeblock, se evidenció que estos kits cubrían las necesidades, y se decidió adquirir varias unidades de ellos. De este modo, se proporciona a cada equipo de alumnos un kit de desarrollo con todas las piezas y herramientas necesarias, de forma que cada equipo pueda llevar a cabo el desarrollo de su robot a lo largo de las diferentes sesiones de prácticas sin necesidad de montar y desmontar el robot en cada sesión.

Finalmente, cabe mencionar que el aula que la universidad y la Escuela Politécnica Superior han facilitado para las prácticas de laboratorio de la asignatura es un aula pensada específicamente para prácticas de robótica, donde cada equipo de alumnos comparte una mesa circular con un espacio de trabajo amplio y adecuado para el montaje de los robots. Además, en el aula hay también hay un espacio dedicado al circuito de pruebas para los robots.

\section{ACTIVIDADES PROPUESTAS}

Al comienzo de la asignatura, los alumnos fueron agrupados en equipos, de forma que los miembros de cada equipo debían colaborar entre ellos para llevar a cabo un pequeño proyecto de robótica para resolver ciertos objetivos marcados en la primera sesión. El proyecto se ha desarrollado a lo largo del semestre, en las 9 sesiones de laboratorio disponibles, y se ha divido en 5 actividades tal y como resume la Tabla 1.

Tabla 1: Actividades y disponibilidad de tiempo.

\begin{tabular}{|l|l|l|}
\hline Actividad & Sesiones & Horas \\
\hline $\begin{array}{l}\text { 1. Planificación y documentación de } \\
\text { un proyecto. }\end{array}$ & 1 & 1,5 \\
\hline 2. Introducción a Arduino & 1 & 1,5 \\
\hline 3. Proyecto de robótica básico & 2 & 3 \\
\hline $\begin{array}{l}\text { 4. Proyecto final para el circuito de } \\
\text { pruebas }\end{array}$ & 4 & 6 \\
\hline $\begin{array}{l}\text { 5. Competición en el circuito de } \\
\text { pruebas }\end{array}$ & 1 & 3 \\
\hline
\end{tabular}

En resumen, el proyecto consiste en el desarrollo de un robot que sea capaz de recorrer un circuito de pruebas a la vez que resuelve ciertas tareas especificadas. Tanto el circuito como las tareas son presentados a los alumnos en la primera sesión de prácticas, de forma que ellos puedan tener en mente los objetivos a lo largo de todo el desarrollo.

Mientras las primeras cuatro actividades pretenden introducir a los alumnos los conceptos y habilidades básicas, la cuarta es la actividad principal de las prácticas, ya que en ella los equipos de alumnos deben desarrollar los robots para una competición final, que conforma la quinta actividad. En la competición, cada equipo debe mostrar como su robot cumple los objetivos en un circuito de pruebas, y como de bien lo hace en comparación con los robots de los otros equipos.

En los siguientes puntos se describe con más detalle en qué consisten las diferentes actividades, cómo se desarrollan y cómo se evalúan las mismas. 
Durante el curso 2015-16, los 62 alumnos matriculados en la asignatura estaban distribuidos en 4 grupos de prácticas de laboratorio. A su vez, en cada grupo de prácticas se organizaron 3 o 4 equipos de alumnos, resultando en un total de 11 equipos compuestos cada uno de 5 o 6 alumnos.

\subsection{Planificación y documentación de un proyecto}

El objetivo de esta primera actividad es introducir a los alumnos las diferentes etapas en las que se divide el desarrollo de un proyecto de ingeniería, además de enseñarles cómo se prepara y documenta adecuadamente un proyecto.

Cada proyecto requiere una primera etapa de planificación y diseño, que debe ser realizada por cada equipo de alumnos de acuerdo a un borrador del robot que planea desarrollar para cumplir los objetivos en el circuito de pruebas. Los alumnos deben afrontar esta etapa usando, además de los conocimientos que ellos ya tienen, los conocimientos que adquieren en las actividades dos y tres, así como otros métodos que ellos pueden investigar.

Las dos siguientes etapas son la implementación y la puesta en marcha del robot, que se desarrollan en las dos últimas actividades.

La evaluación de esta primea actividad se realiza mediante un breve informe que cada equipo debe entregar a los profesores antes de comenzar la actividad 4. Este informe debe describir las características básicas del diseño propuesto para el robot, los fundamentos de los métodos usados en el mismo, las fuentes bibliográficas de dónde se han obtenido los métodos, y una planificación de los recursos (personas y materiales) y tiempo disponibles. Los profesores evalúan los informes con las propuestas e indican a los equipos los aspectos que deberían ser corregidos para que pueden lograr un robot funcional.

\subsection{Introducción a Arduino}

Puesto que el controlador Orion usado con los robots está basado en Arduino, es necesario introducir esta plataforma a los alumnos. Este es el objetivo de esta segunda actividad.

Por una parte, se enseña a los alumnos como descargar, instalar, configurar y usar el software necesario, que básicamente consiste en el IDE de Arduino y en la biblioteca de clases proporcionada por Makeblock para programar más fácilmente los módulos de sus kits. Por otra parte, se introduce al alumno los fundamentos de la programación de Arduino con $\mathrm{C}++$. Esto incluye el uso de las funciones "setup" y "loop" de que consta todo programa de Arduino [2], así como la creación de funciones para gobernar los accionamientos en base a la información de los módulos de sensores, y los comandos de decisión que proporcionan la lógica necesaria al programa. Más concretamente, en esta actividad, los equipos de alumnos deben desarrollar un programa para gobernar los dos motores CC, primero de acuerdo a unas temporizaciones determinadas, y después según la distancia que proporciona el módulo con el sensor de ultrasonidos.

Para la evaluación de esta actividad, los profesores comprueban el funcionamiento de los programas que los distintos equipos han desarrollado, mientras éstos explican cómo funcionan sus programas.

\subsection{Proyecto de robótica básico}

Esta actividad tiene como objetivo introducir a los alumnos el kit de construcción de robots, de forma que ellos se familiaricen con las piezas y herramientas disponibles, y adquieran las habilidades necesarias para el montaje de robots. Para esto, los equipos de alumnos tienen que montar y poner en marcha un robot móvil básico, compuesto de dos cadenas movidas por dos motores de CC, el controlador Orion, el conjunto de pilas, el módulo con sensor de ultrasonidos y el módulo de comunicación Bluetooth, además de las estructuras metálicas necesarias.

El robot a montar, que es mostrado en la Figura 3, puede ser ensamblado siguiendo una guía de Makeblock. Después de montar el robot, los equipos deben probarlo usando una aplicación para teléfonos inteligentes que conecta con el controlador mediante Bluetooth y permite operar remotamente el robot.

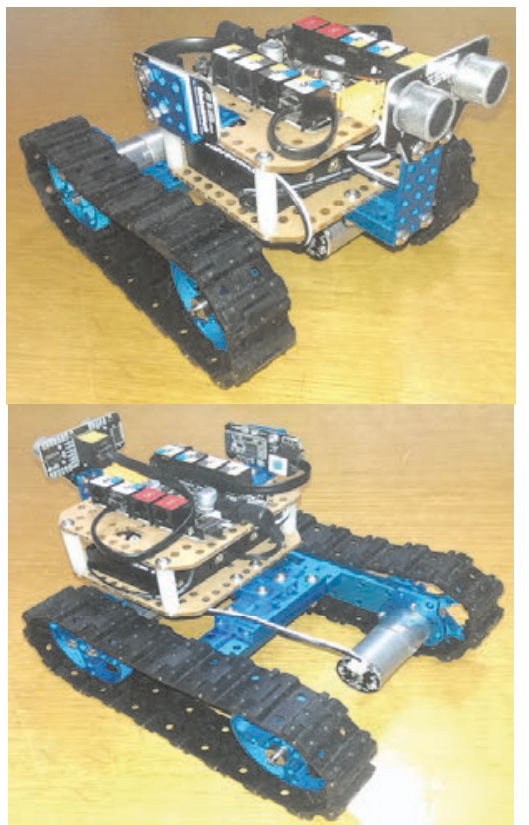

Figura 3: Robot básico construido en la actividad 3. 


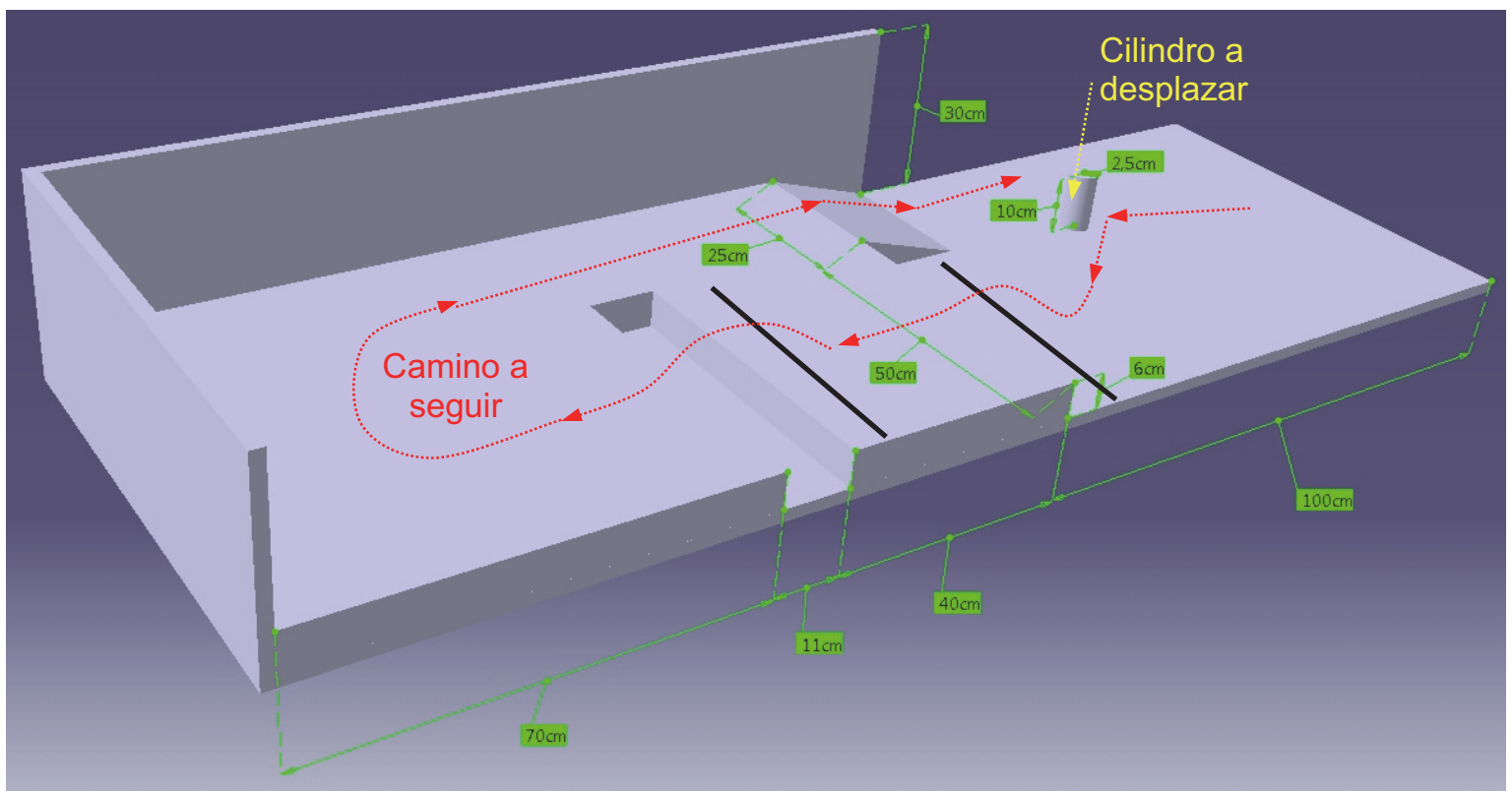

Figura 4: Representación del circuito de pruebas.

Finalmente los equipos deben diseñar su propio programa para el controlador, de forma que el robot se mueva de forma autónoma por el suelo mientras evita los posibles obstáculos detectados por el sensor de ultrasonidos.

La evaluación de esta actividad consiste en que los profesores comprueben cómo están ensamblados y cómo funcionan los robots, mientras los equipos explican cómo funciona el programa que han implementado en el controlador.

\subsection{Proyecto final para el circuito de pruebas}

La actividad principal de las prácticas de laboratorio es el proyecto que debe ser llevado a cabo por cada equipo de alumnos. Más concretamente, cada equipo debe diseñar, construir y programar un robot móvil que sea capaz de seguir un camino y resolver unas tareas simples en un circuito de pruebas.

La distribución y las medidas del circuito de pruebas se proporcionan a los alumnos antes de esta actividad, de forma que los equipos puedan pensar en el diseño del robot durante el desarrollo las actividades previas, y ellos también puedan preparar el informe para la primera actividad. La Figura 4 muestra una representación 3D del circuito de pruebas, donde se ha marcado con una línea roja el camino que deben seguir los robots.

Las tareas concretas que deben llevar a cabo los robots en el circuito son las siguientes:

- Comenzar desde la parte derecha del circuito, en una posición elegida por cada equipo.

- Mover hacia adelante hasta detectar el cilindro, y desplazar el cilindro a un lado, fuera del camino.
- Avanzar hasta el escalón, que está señalado con una línea negra en el suelo antes del mismo, y subir al nivel superior del circuito.

- Mover hacia adelante hasta el foso, que está señalado con una línea negra antes del mismo, y atravesar el foso sin tocar el fondo del mismo.

- Mover hacia adelante, y antes de chocar con la pared, girar $180^{\circ}$ a la derecha.

- Avanzar pasando sobre el puente y bajar por la rampa para volver al lado derecho del circuito, sin chocar con la pared.

Las dimensiones de las partes del circuito son tales que, para resolver las diferentes tareas, no basta con diseñar un vehículo con ruedas o cadenas. El robot necesita sensores y accionamientos adicionales, como por ejemplo el sensor de ultrasonidos para detectar el cilindro, la pinza para agarrarlo y sacarlo del camino, el sensor de infrarrojos para detectar las líneas en el suelo, o un brazo frontal con una rueda adicional para superar el foso. El kit de robótica contiene todos esos elementos y más, pero cada equipo de alumnos es quién decide que componentes debe tener su robot y como son usados éstos. En la Figura 5 se puede ver a uno de los equipos montando su robot en el laboratorio.

Esta actividad no se evalúa como tal, sino que el funcionamiento del robot en el circuito se evalúa mediante la competición descrita a continuación.

\subsection{Competición en el circuito de pruebas}

La competición se planifica en la última sesión de prácticas de laboratorio, antes del periodo de exámenes. 


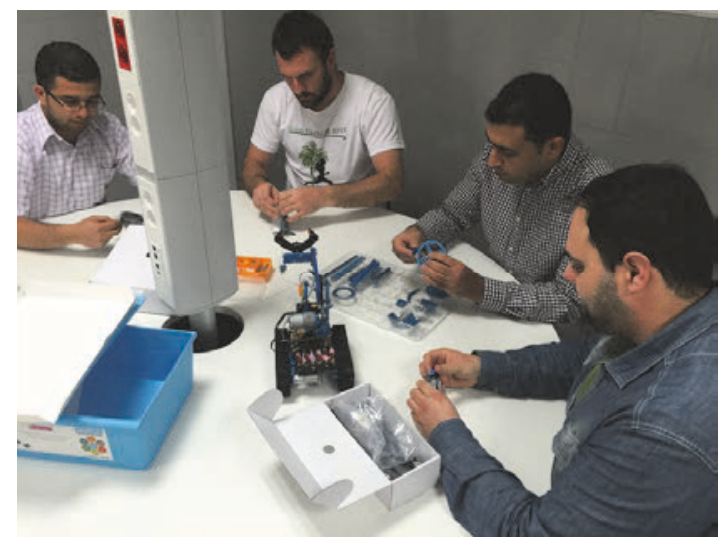

Figura 5: Uno de los equipos montando su robot en el laboratorio.

Todos los equipos de alumnos deben competir entre ellos para mostrar cómo sus robots completan el circuito de pruebas con el mejor tiempo. Para la determinar la puntuación de la actividad, además de considerar el tiempo total del recorrido, los profesores tienen en cuenta los siguientes aspectos: la originalidad del diseño, la distribución adecuada de sus elementos, el uso de diferentes sensores y accionamientos, cómo de bien resuelve el robot cada tarea, la originalidad en la forma de resolver las tareas, la claridad del programa del controlador, y la idoneidad de los algoritmos utilizados.

En la Figura 6-A se puede ver 4 de los 11 robots construidos por los equipos de alumnos durante el curso 2015-16. Los alumnos han diseñado y montado robots con estructuras muy dispares para superar los objetivos del circuito de pruebas. La Figura 6-B muestra momentos clave de algunos robots durante la competición: apartando el cilindro con una pinza, subiendo el escalón gracias a un brazo que actúa de palanca, cruzando el foso gracias a unas ruedas anteriores, y girando $180^{\circ}$ sin chocar con la pared usando el sensor de ultrasonidos.
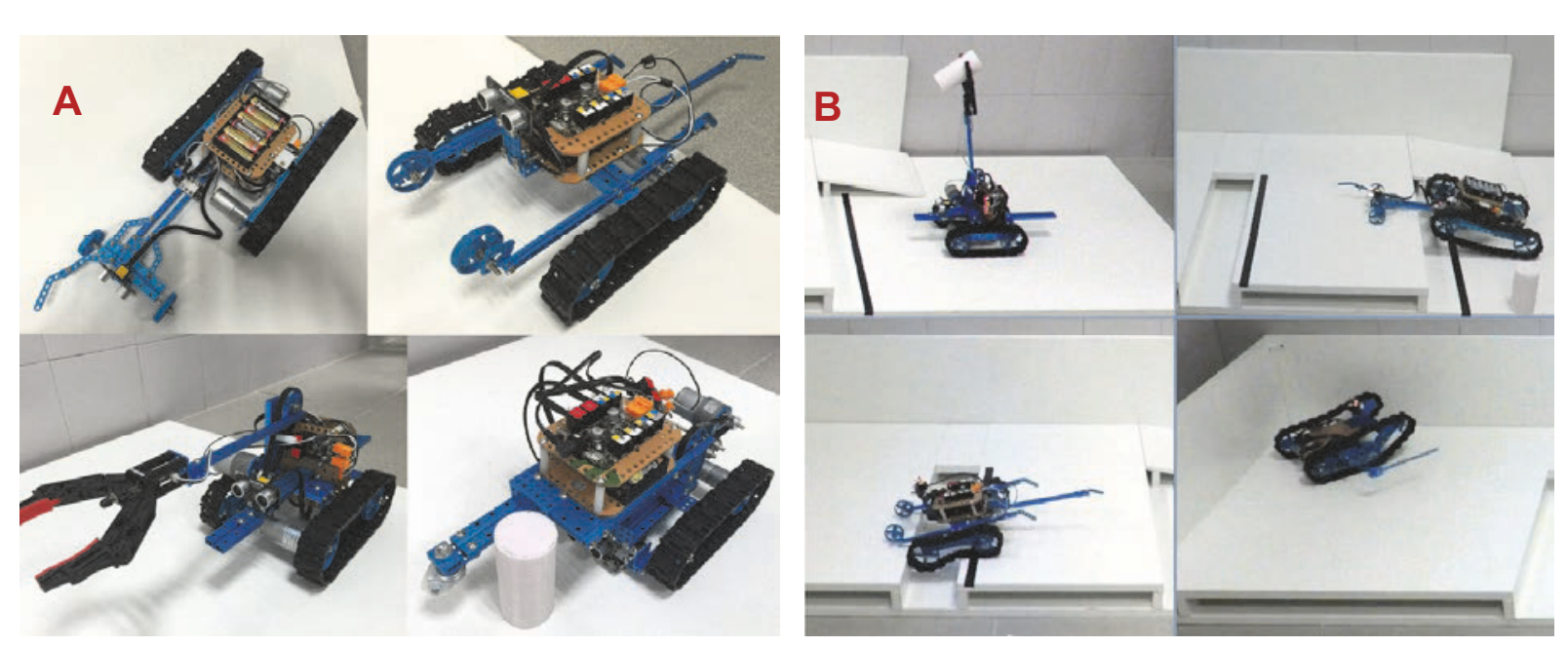

Figura 6: A) Cuatro de los robots montados por los alumnos. B) Instantáneas de algunos robots durante la competición en el circuito de pruebas.

\section{RESULTADOS}

Cabe destacar que todos los alumnos que comenzaron las prácticas de laboratorio han continuado realizando las mismas hasta el final, y han participado en la competición. Además, la asistencia al laboratorio ha sido muy alta; de los 62 alumnos matriculados, solo 4 no han realizado las prácticas. Todos los equipos han querido aprovechar cada hora de laboratorio para construir su robot, y la implicación de los alumnos ha sido muy alta.

La Tabla 2 muestra un resumen de los resultados de la competición. En ella se indica el mejor tiempo (MT) que cada robot ha tardado en completar todo el circuito (se muestra un guion si no se completó), y como el robot ha superado los principales objetivos.

Tabla 2: Principales resultados de la competición.

\begin{tabular}{|c|c|c|c|c|c|}
\hline Equipo & MT & Cilindro & Escalón & Foso & Giro \\
\hline 1 & $27 \mathrm{~s}$ & $\begin{array}{l}\text { Aparta con } \\
\text { brazo }\end{array}$ & $\begin{array}{l}\text { Brazo- } \\
\text { palanca }\end{array}$ & $\begin{array}{l}\text { Brazo- } \\
\text { palanca }\end{array}$ & $\begin{array}{c}\text { Por } \\
\text { tiempo }\end{array}$ \\
\hline 2 & - & Pinza & $\begin{array}{l}\text { Cadenas y } \\
\text { velocidad }\end{array}$ & $\begin{array}{l}\text { Apoya } \\
\text { pinza }\end{array}$ & - \\
\hline 3 & - & - & $\begin{array}{l}\text { Cadenas y } \\
\text { velocidad }\end{array}$ & - & - \\
\hline 4 & $22 s$ & $\begin{array}{c}\text { Desplaza con } \\
\text { brazo }\end{array}$ & $\begin{array}{l}\text { Cadenas y } \\
\text { velocidad }\end{array}$ & $\begin{array}{l}\text { Cadenas y } \\
\text { velocidad } \\
\end{array}$ & $\begin{array}{c}\text { Sensor } \\
\text { US }\end{array}$ \\
\hline 5 & $38 \mathrm{~s}$ & - & $\begin{array}{l}\text { Cadenas y } \\
\text { velocidad }\end{array}$ & $\begin{array}{l}\text { Cadenas y } \\
\text { velocidad }\end{array}$ & $\begin{array}{c}\text { Por } \\
\text { tiempo }\end{array}$ \\
\hline 6 & $30 \mathrm{~s}$ & $\begin{array}{c}\text { Desplaza con } \\
\text { gancho }\end{array}$ & $\begin{array}{l}\text { Cadenas y } \\
\text { velocidad }\end{array}$ & $\begin{array}{l}\text { Cadenas y } \\
\text { velocidad }\end{array}$ & $\begin{array}{c}\text { Sensor } \\
\text { US }\end{array}$ \\
\hline 7 & - & $\begin{array}{c}\text { Desplaza con } \\
\text { brazo }\end{array}$ & - & - & - \\
\hline 8 & $31 \mathrm{~s}$ & - & Cadenas & $\begin{array}{c}\text { Apoya en } \\
\text { la pinza }\end{array}$ & $\begin{array}{c}\text { Por } \\
\text { tiempo }\end{array}$ \\
\hline 9 & $42 s$ & $\begin{array}{c}\text { Desplaza con } \\
\text { una cola }\end{array}$ & $\begin{array}{l}\text { Cadenas y } \\
\text { velocidad }\end{array}$ & $\begin{array}{c}\text { Ruedas } \\
\text { auxiliares }\end{array}$ & $\begin{array}{c}\text { Sensor } \\
\text { US }\end{array}$ \\
\hline 10 & - & Pinza & $\begin{array}{c}\text { Pinza como } \\
\text { palanca }\end{array}$ & $\begin{array}{l}\text { Cadenas y } \\
\text { velocidad }\end{array}$ & - \\
\hline 11 & $28 s$ & $\begin{array}{l}\text { Desplaza con } \\
\text { una cola }\end{array}$ & $\begin{array}{l}\text { Cadenas y } \\
\text { velocidad }\end{array}$ & $\begin{array}{c}\text { Rueda } \\
\text { auxiliar }\end{array}$ & $\begin{array}{c}\text { Sensor } \\
\text { US }\end{array}$ \\
\hline
\end{tabular}


La mayoría de alumnos tenía un nivel bastante bajo de programación cuando empezó la asignatura. Pero gracias a la facilidad de Arduino, todos los equipos pudieron programar su robot. Sin embargo, fueron bastantes los equipos que resolvieron muchas tareas del circuito mediante temporización, en vez de hacer un mejor uso de los sensores disponibles. Esto causó que varios equipos tuvieran problemas cuando las baterías de sus robots no tenían su máxima carga, y en consecuencia esos robots no se movían a la velocidad que habían usado en las pruebas, y por eso fallaba la precisión de las temporizaciones.

El método más usado para apartar el cilindro ha sido mediante un brazo delantero o una cola trasera que desplazaba el cilindro mientras el vehículo giraba, si bien hubo dos robots que usaron la pinza. La mayoría de robots superaron el escalón mediante "fuerza bruta", gracias a la tracción por cadenas y a un avance a gran velocidad. Hubo dos equipos que superaron el escalón con un brazo articulado usado como palanca. Los métodos usados para cruzar el foso han sido más variados: muchos robots usaron la velocidad y un buen diseño de las cadenas, pero también se usaron ruedas delanteras auxiliares o un brazo como palanca. Cuatro robots consiguieron realizar el giro de $180^{\circ}$ correctamente usando el sensor de ultrasonidos para no chocar con las paredes. Se pude decir que muchos equipos optaron por soluciones sencillas para superar los objetivos.

Hubo equipos cuyo robot no completó todo el circuito. Pero como para la evaluación también se consideró el desarrollo de las actividades 1 a 4 , así como la asistencia y participación en las sesiones, todos los alumnos con asistencia regular han superado las prácticas. Aunque en la actividad de la competición las notas abarcan desde 3 hasta 10 sobre 10 , en las actividades previas las notas fueron bastante buenas. Los informes de los proyectos de todos los equipos sobre la planificación y diseño inicial de su robot fueron correctos o buenos, y no fue necesario indicar mejoras o correcciones del diseño a ningún equipo antes de la actividad 4 .

Varios equipos solicitaron disponer de más tiempo de prácticas para poder concluir los diseños que tenían previstos antes de la competición. Aprovechando que había disponibilidad de fechas, se optó finalmente por añadir una sesión de prácticas de laboratorio adicional para la actividad 4 para todos los grupos de prácticas.

También cabe mencionar que hubo bastante competitividad entre equipos en el desarrollo de la asignatura. Los alumnos llegaron a pedir que se suministrase una caja a cada equipo para ocultar los robots cuando estos se guardaban en los armarios entre las sesiones.

\section{CONCLUSIONES}

Atendiendo a la asistencia a las sesiones de prácticas laboratorio, las notas de las actividades, y a la participación en la competición, se puede concluir que la gran mayoría de los alumnos ha mostrado un gran interés por desarrollar las actividades propuestas. Se puede decir que la elección de las actividades basadas en el proyecto de construcción de un robot pensando en una competición final ha resultado ser un éxito.

El hecho de que el controlador del robot sea una versión mejorada de la plataforma Arduino, con un método de conexionado del cableado muy sencillo, ha contribuido a los resultados mencionados, gracias a que los alumnos pueden poner en marcha esta plataforma y aprender a programarla rápidamente. Es más, los alumnos se centran en los verdaderos problemas de las actividades planteadas, como son el desarrollo y la programación de los robots, en vez de problemas relacionados con la instalación o configuración del software y el hardware, que prácticamente no se han dado en las prácticas presentadas aquí.

Finalmente, cabe decir que hemos encontrado que el kit Ultimate Robot ha resultado ser sencillo de poner en marcha y utilizar, así como muy robusto y bastante fiable, y hemos quedado muy satisfechos con este kit.

\section{Agradecimientos}

Queremos agradecer la financiación y el apoyo de la Escuela Politécnica Superior de la Universidad de Alicante, así como de la Generalitat Valenciana a través del proyecto PROMETEO/2013/085.

\section{Referencias}

[1] Arduino Home (2016). On-line, consulatdo en junio de 2016: http://www.arduino.cc.

[2] Banzi, M. (2011). Getting Started with Arduino. 2nd Edition. USA: O'Reilly.

[3] Candelas, F.A., Garcia, G.J., Puente, S.T., Pomares, J., Jara, C.A., Alepuz, J., Mira, D., Torres, F. (2015), "Experiences on Using Arduino for Laboratory Experiments of Automatic Control and Robotics". In Proc. 3rd IFAC Workshop on Internet Based Control Education (IBCE 2015). Brescia, Italy.

[4] CEA (2016), "Curso práctico on-line de Arduino Avanzado". On-line, consultado en junio de 2016:

http://intranet.ceautomatica.es/curso-online-decea-curso-practico-line-de-arduino-avanzado 
[5] Guzmán, J. L., Dormido, S., Berenguel. M. (2013), "Interactivity in education: An experience in the automatic control field", Computer Applications in Engineering Education, 27, 2, pp. 360-371.

[6] Makeblock. On-line, consulatdo en junio de 2016: http://learn.makeblock.cc/ultimate-robotkit/

[7] Makeblock (2016), Orion. On-line, consultado en junio de 2016:

http://learn.makeblock.com/makeblock-orion/

[8] mBlock (2016). On-lime, consultado en junio de 2016): http://www.mblock.cc/

[9] Pérula-Martínez, R., García-Haro, J.M., Balaguer, C. Salichs, M.A. (2016), Developing Educational Printable Robots to Motivate University Students Using Open Source Technologies. Journal of Intelligent and Robotic Systems, vol. 81 (1), pp.25-39.

[10] Sobota, J., Pišl, R., Balda, P., Schlegel, M. (2013), "Raspberry Pi and Arduino Boards in Control Education (I)". In Proc. 10th IFAC Symposium on Advances in Control Education (ACE2013). Sheffield, UK.

[11] Universidad de Alicante (2016), "Grado en Ingeniería Robótica”. On-line, consultado en junio de 2016:

http://cvnet.cpd.ua.es/webcvnet/planestudio/pla nestudiond.aspx? plan $=\mathrm{C} 211 \&$ caca $=2015$ -

16\&lengua $=\mathrm{E}$

[12] Valera, A., Soriano, A., Vallés, M. (2014) "Low-Cost Platforms for Realization of Mechatronics and Robotics Practical Works". Revista Iberoamericana de Automática e Informática Industrial (RIAI), Elsevier, vol. 11 (4), pp. 363-376.

[13] Warren, J.D. Adams, J., Molle, H. (2011), Arduino Robotics (Technology in Action). USA: Apress. 